\title{
The Theoretical Foundation in the Holder Space of Quadratures Method of Solving Nonlinear Singular Integral Equations Given on Closed Smooth Contour
}

\author{
Omar M. Hawamdeh ${ }^{1}$ \& Adeeb A. Alrahmneh ${ }^{2}$
}

\author{
${ }^{1}$ Mathematics, Al-Balqa Applied University \\ ${ }^{2}$ Applied Statistics, Al-Balqa Applied University \\ omar_h1964@yahoo.com \\ Received: April 25, 2012 Accepted: May 8, 2012 Online Published: July 10, 2012 \\ doi:10.5539/jmr.v4n4p74 URL: http://dx.doi.org/10.5539/jmr.v4n4p74
}

Correspondence: Omar M. Hawamdeh, Assistant Professor in Mathematics, Al-Balqa Applied University. E-mail:

\begin{abstract}
A theoretical foundation is proposed for some computing schemes of quadratures method for solving nonlinear singular integral equations (SIE) given on arbitrary closed smooth contour. Estimations of convergence rate of approximative solution are obtained in Holder spaces.
\end{abstract}

Keywords: smooth closed contour, quadratures method, Holder Spaces, nonlinear singular integral equations

\section{Introduction}

In the case of more smooth contours of integration $\Gamma$, namely, contours from class $C(2, v)$ the un improvable estimates of convergence rate of method are proved. Convergence of quadratures methods for nonlinear SIE given on arbitrary closed smooth contours.

Let $\Gamma$ be a closed smooth contour (Mushelishvili, 1968) bounding a simple connected region $F+$ of the complex plane $C$ containing the point $t=0$. In the Banach space of functions $H_{\beta}(\Gamma)$ (Mushelishvili, 1968) satisfying on $\Gamma$ the Holder condition with the exponent $\beta(0<\beta<1)$ consider a nonlinear SIE

$$
A(\varphi) \equiv \Phi\left[t ; \varphi(t) ; S_{\tau} h(t, \tau ; \varphi(\tau))\right]=f(t)
$$

where $\Phi[t ; u ; v](t \in \Gamma ;|u|,|v|<\infty), h(t, \tau ; u)(t, \tau \in \Gamma ;|u|<\infty)$ and $f(t)$ are known continuous functions of their arguments, the singular integral

$$
S_{\tau} h(t, \tau ; \varphi(\tau)) \equiv \frac{1}{\pi i} \int_{\Gamma} \frac{h(t, \tau ; \varphi(\tau))}{\tau-t} d \tau, t \in \Gamma,
$$

is understood in the meaning of Cauchy principal value, and $\varphi(t)(t \in \Gamma)$ is an unknown function.

The researchers seek for the approximate solution of nonlinear SIE (1) as a polynomial

$$
\varphi_{n}(t)=\sum_{j=0}^{2 n} c_{j} l_{j}(t),(t \in \Gamma)
$$

where (Zolotarevschi, 1991; Scichiuc, 1984)

$$
l_{j}(t)=\prod_{k=0}^{2 n}\left(\frac{t-t_{k}}{t_{j}-t_{k}}\right)\left(\frac{t_{j}}{t}\right)^{n} \equiv \sum_{k=-n}^{n} A_{k}^{(j)} t^{k}, j=\overline{0,2 n}, t \in \Gamma .
$$




\section{Method}

For quadratures methods the unknown coefficients $\left\{c_{j}\right\}_{j=0}^{2 n}$ will be determinated from the system of nonlinear equations (SNE)

$$
\Phi\left[t_{j} ; c_{j} ; \sum_{k=0}^{2 n} h\left(t_{j}, t_{k} ; c_{k}\right) t_{k}^{p}\left(\sum_{v=p}^{n} A_{v}^{(k)} t_{j}^{\nu-p}-\sum_{v=-n}^{p-1} A_{v}^{(k)} t_{j}^{v-p}\right)\right]=f\left(t_{j}\right), j=\overline{0,2 n}, p=0 ; 1,
$$

where $\left\{t_{j}\right\}_{j=0}^{2 n}$ is a set of pairwize distinct points on $\Gamma$.

Scichiuc note that the foundation of quadratures method for the equation (1) is obtained for the case of Lyapunov's contour $\Gamma$ and $p=0$ in the computing scheme (3) (Scichiuc, 1984). In present work the following results are obtained for the class of closed smooth contours $\Gamma$ and $p=1$ in SNE (3). In this case the convergence rate of approximative solution is improved.

Theorem 1 Let the following conditions be fulfilled:

1) $\Phi_{u^{2}}^{\prime \prime}[t ; u ; v], \Phi_{v^{2}}^{\prime \prime}[t ; u ; v], \Phi_{u v}^{\prime \prime}[t ; u ; v] \in H_{\alpha ; 1 ; 1}(\Gamma), h_{u^{2}}^{\prime \prime}(t, \tau ; u) \in H_{\mu ; \alpha ; 1}(\Gamma),(0<\alpha<\mu<1)$;

2) The nonlinear SIE (1) in some sphere of the space $H_{\beta}(\Gamma)(0<\beta<\alpha \leq 1)$ has a unique solution $\varphi(t) \in H_{\alpha}^{r}(\Gamma), r=$ $0,1, \ldots$;

3) $C_{\varphi}^{2}(t)-D_{\varphi}^{2}(t) \neq 0(t \in \Gamma)$, ind $\left[\left(C_{\varphi}(t)+D_{\varphi}(t)\right)\left(C_{\varphi}(t)-D_{\varphi}(t)\right)^{-1}\right]=0(t \in \Gamma)$, where

$$
\begin{gathered}
C_{\varphi}(t)=\Phi_{u}^{\prime}\left[t ; \varphi(t) ; S_{\tau} h(t, \tau ; \varphi(\tau))\right] \in H_{\alpha ; 1 ; 1}^{r}(\Gamma), \\
D_{\varphi}(t)=\Phi_{v}^{\prime}\left[t ; \varphi(t) ; S_{\tau} h(t, \tau ; \varphi(\tau))\right] h_{u}^{\prime}(t, t ; \varphi(t)) \in H_{\alpha ; 1 ; 1}^{r}(\Gamma), t \in \Gamma .
\end{gathered}
$$

In cases $r=0$ or $r=1$ conditions (4) are fulfilled by 1 );

4) $\operatorname{dim} \operatorname{Kern} A^{\prime}(\varphi)=0$, where $A^{\prime}$ is defined in the following way: $\forall \varphi^{0}(t) \in H_{\beta}(\Gamma)$,

$$
\begin{aligned}
& \left(A^{\prime}\left(\varphi^{0}\right) g\right)(t) \equiv \Phi_{u}^{\prime}\left[t ; \varphi^{0}(t) ; S_{\tau} h\left(t, \tau ; \varphi^{0}(\tau)\right)\right] g(t)+\Phi_{v}^{\prime}\left[t ; \varphi^{0}(t) ; S_{\tau} h\left(t, \tau ; \varphi^{0}(\tau)\right)\right] S_{\tau}\left[h_{u}^{\prime}\left(t, \tau ; \varphi^{0}(\tau)\right) g(\tau)\right] \\
= & C_{\varphi^{0}}(t) g(t)+D_{\varphi^{0}}(t) S_{\tau} g(\tau)+\frac{1}{\pi i} \int_{\Gamma} \Phi_{v}^{\prime}\left[t ; \varphi^{0}(t) ; S_{\tau} h\left(t, \tau ; \varphi^{0}(\tau)\right)\right] \frac{h_{u}^{\prime}\left(t, \tau ; \varphi^{0}(\tau)\right)-h_{u}^{\prime}\left(t, t ; \varphi^{0}(t)\right)}{\tau-t} g(\tau) d \tau ;
\end{aligned}
$$

5) The points $t_{j}(j=\overline{0,2 n})$ form on $\Gamma$ a system of Fejer's nodes (Smirnov, \& Lebedev, 1964, p. 36), i. e.,

$$
t_{j}=\psi\left(w_{j}\right), w_{j}=\exp \left(\frac{2 \pi i}{2 n+1}(j-n)\right), i^{2}=-1, j=\overline{0,2 n},
$$

$t=\psi(w)$ is the Riemann's function which realizis the conform mapping of exterior of the unitary circumfirence $\Gamma_{0}(=|W|=1)$ on the exterior of $F^{+} U \Gamma$ such that $\psi(\infty)=\infty, \psi^{\prime}(\infty)=$ const $>0$.

Then there exists a $2 n+1$ dimensional point $\left\{y_{k}\right\}_{k=-n}^{n}$, in an neighbourhood of which SNE (3) has a unique solution $\left\{c_{j}\right\}_{j=0}^{2 n}$ for all $n$ begining with a certain one. The approximate solutions (2) converge in the norm of $H_{\beta}(\Gamma)$ to $\varphi(t)$ as narrow $\infty$ for any function $f(t) \in H_{\alpha}^{r}(\Gamma)$. For the rate of convergence the following estimation holds

$$
\left\|\varphi-\varphi_{n}\right\|_{\beta}=O\left(n^{-r+\beta-\sigma(\alpha)} \ln ^{2} n\right) H\left(\varphi^{(r)} ; \sigma(\alpha)\right),
$$

where $\sigma(\alpha)=\alpha$, if $0<\alpha<1$, and $\sigma(\alpha)=\alpha-\varepsilon(\forall \varepsilon>0)$, if $\alpha=1$.

Proof. By conditions 1) of the theorem 1 the nonlinear operator A, determinated by the left-hand side of the equation (1) is Freshet differenti able (Gabdullaev \& Gorlov, 1976) at every point $\varphi^{0}$ of $H_{\beta}(\Gamma)(0<\beta<\alpha \leq 1)$ and its derivative has the form described by condition 4) of the theorem. Moreover as long as the Singular operator $S_{\tau}$ is bounded in space $H_{\beta}(\Gamma)$, in the sphere $\left\|g-\varphi^{0}\right\|_{\beta} \leq r(r>0)$ of $H_{\beta}(\Gamma)$ the linear operator $A^{\prime}$ satisfies the Lipshitz condition

$$
\left\|A^{\prime}\left(g_{1}\right)-A^{\prime}\left(g_{2}\right)\right\|_{\beta} \leq L\left\|g_{1}-g_{2}\right\|_{\beta},
$$

where $L$ is a completely definite constant, its value depends on $r$, on element $\varphi^{0}(t) \in H_{\beta}(\Gamma)$ and on functions $\Phi$ and $h$. 
Let $X(t)$ be an arbitrary continuous on $\Gamma$ function and

$$
U_{n}(x)=U_{n}(X, t)=\sum_{j=0}^{2 n} X\left(t_{j}\right) l_{j}(t)
$$

its interpolating Lagrange polynomial, constructed by nodes $\left\{t_{j}\right\}_{j=0}^{2 n} \subset \Gamma$. Then SNE (3) can be written as an equation.

$$
\left(B_{n, p}\left(\varphi_{n}\right)\right) \equiv U_{n} \Phi\left[t ; \varphi_{n}(t) ; S_{\tau}\left[\frac{1}{\tau^{p}} U_{n}^{\tau}\left(\tau^{p} h\left(t, \tau ; \varphi_{n}(\tau)\right)\right)\right]\right]=U_{n} f(t), p=0 ; 1, t \in \Gamma,
$$

where $B_{n, p}$ is a nonlinear operator on the subspace $X n$ of polynomial functions of the form (2) with the same norm as in $H_{\beta}(\Gamma)$. By the same conditions 1) of the theorem, the operator $B_{n, p}$ from (7) is also Freshet differentiable at every point $\varphi_{n}^{0}$ of $X n$ and its derivative has the form

$$
\begin{gathered}
\left(B_{n, p}^{\prime}\left(\varphi_{n}^{0}\right) g_{n}\right) \equiv U_{n}\left\{\Phi_{u}^{\prime}\left[t, \varphi_{n}^{0}(t) ; S_{\tau}\left[\frac{1}{\tau^{p}} U_{n}^{\tau}\left(\tau^{p} h\left(t, \tau ; \varphi_{n}^{0}(\tau)\right)\right)\right]\right] g_{n}(t)\right\}+ \\
+U_{n}\left\{\Phi_{v}^{\prime}\left[t ; \varphi_{n}^{0}(t) ; S_{\tau}\left[\frac{1}{\tau^{p}} U_{n}^{\tau}\left(\tau^{p} h\left(t, \tau ; \varphi_{n}^{0}(\tau)\right)\right)\right]\right] S_{\tau}\left[\frac{1}{\tau^{p}} U_{n}^{\tau}\left(\tau^{p} h_{u}^{\prime}\left(t, \tau ; \varphi_{n}^{0}(\tau)\right)\right) g_{n}(\tau)\right]\right\}
\end{gathered}
$$

Now let the nodes $\left\{t_{j}\right\}_{j=0}^{2 n} \subset \Gamma$ be computed in accordance with condition 5) of the theorem.

Then (Zolotarevschi, 1991)

$$
\left\|U_{n}\right\|_{\beta} \leq \mu_{1}+\mu_{2} \ln (2 n+1),
$$

where, here and later on, $\mu_{k}(k=1,2, \ldots)$ are completely definite constants not depending on $n$. Hence, using (9), the linear operator $B_{n, p}^{\prime}\left(\varphi_{n}^{0}\right)$ defined in (8) satisfies Lipschitz condition (6) with the new constant.

$$
\left\|B_{n, p}^{\prime}\left(z_{n}^{(1)}\right)-B_{n, p}^{\prime}\left(z_{n}^{(2)}\right)\right\|_{\beta} \leq\left(\mu_{3}+\mu_{4} \ln (2 n+1)+\mu_{5} \ln ^{2}(2 n+1)\right)\left\|z_{n}^{(1)}-z_{n}^{(2)}\right\|_{\beta}
$$

Let $\varphi_{n}^{*}(t)\left(\in X_{n}(\Gamma)\right)$ be the best uniform approximation polynomial of $\varphi(t)$. Then from (6) and lemma 6.1 (Zolotarevschi, 1991) there we get (Seichiuc, 1997)

$$
\left\|A^{\prime}(\varphi)-A^{\prime}\left(\varphi_{n}^{*}\right)\right\|_{\beta} \leq \frac{\mu_{6}}{n^{r+\sigma(\alpha)-\beta}} H\left(\varphi^{(r)} ; \sigma(\alpha)\right) .
$$

Hence from the reversibility of $A^{\prime}(\varphi)$ and from Banach theorem it follows that for all $n\left(n \geq n_{1}\right)$ such that

$$
\frac{\mu_{6}}{n^{r+\sigma(\alpha)-\beta}} H\left(\varphi^{(r)} ; \sigma(\alpha)\right)\left\|\left[A^{\prime}(\varphi)\right]^{-1}\right\|_{\beta}=d_{n} \leq q<1
$$

The operator $A^{\prime}\left(\varphi_{n}^{*}\right)$ is also reversible in $H_{\beta}(\Gamma)$, and

$$
\left\|\left[A^{\prime}\left(\varphi_{n}^{*}\right)\right]^{-1}\right\|_{\beta} \leq \frac{\left\|\left[A^{\prime}(\varphi)\right]^{-1}\right\|_{\beta}}{1-d_{n}} \leq \frac{\left\|\left[A^{\prime}(\varphi)\right]^{-1}\right\|_{\beta}}{1-q}=\mu_{7} .
$$

By the theorem about the continuity of the function index (Gahov, 1977) changing there $\varphi$ on $\varphi_{n}^{*}$, the conditions 3 )-4) of the theorem are fulfilled, as $n \geq n_{1}$. From (8) it follows that the operator $B_{n, p}^{\prime}\left(\varphi_{n}^{*}\right)$ is the operator of quadratures method for the operator $A^{\prime}\left(\varphi_{n}^{*}\right)$. Thus for the operators $A^{\prime}\left(\varphi_{n}^{*}\right)$ and $B_{n, p}^{\prime}\left(\varphi_{n}^{*}\right)$ all the conditions of the theorem 8.2 (Zolotarevschi, 1991) are fulfilled. Therefore for sufficiently large $n\left(n \geq n_{2} \geq n_{1}\right)$ the operator $B_{n, p}^{\prime}\left(\varphi_{n}^{*}\right)$ is reversible in $X n(\Gamma)$ and the estimation

$$
\left\|\left[B_{n, p}^{\prime}\left(\varphi_{n}^{*}\right)\right]^{-1}\right\|_{\beta} \leq \mu_{8}
$$

holds. From the estimations (10) and (11) it follows that for sufficiently small $r_{1}$-the inequality

$$
\sup _{\left\|\Psi_{n}-\varphi_{N}^{*}\right\|_{\beta \leq r 1}}\left\|\left[B_{n, p}^{\prime}\left(\varphi_{n}^{*}\right)\right]^{-1}\left[B_{n, p}^{\prime}\left(\Psi_{n}\right)-B_{n, p}^{\prime}\left(\varphi_{n}^{*}\right)\right]\right\| \leq \mu_{8}\left(\mu_{3}+\mu_{4} \ln (2 n+1)+\mu_{5} \ln ^{2}(2 n+1)\right) r_{1}=q<1
$$

holds, where $\psi_{n} \in X_{n}$. 
Let $f(t) \in H_{\alpha}^{r}(\Gamma)(0<\beta<\alpha \leq 1)$. Using the estimations (9), (11), the well known formula of finite increments for operators the inequality $\left\|S_{\tau}\right\|_{\beta} \leq \mu_{9}$ and Lemma 6.1 (Zolotarevschi, 1991, p. 55). We find that for all $n$ begining with a certain one $\left(n \geq n_{3} \geq n_{2}\right)$ the following in equality is true:

$$
\begin{aligned}
& \rho_{n} \equiv\left\|\left[B_{n, p}^{\prime}\left(\varphi_{n}^{*}\right)\right]^{-1}\left[B_{n, p}\left(\varphi_{n}^{*}\right)-U_{n} f\right]\right\|_{\beta}=\left\|\left[B_{n, p}^{\prime}\left(\varphi_{n}^{*}\right)\right]^{-1}\left[B_{n, p}\left(\varphi_{n}^{*}\right)-B_{n, p}(\varphi)\right]\right\|_{\beta} \\
& \leq \mu_{8}\left(\mu_{1}+\mu_{2} \ln (2 n+1)\right)\left\|\Phi\left[t ; \varphi_{n}^{*}(t) ; S_{\tau}\left[\frac{1}{\tau^{p}} U_{n}^{\tau}\left(\tau^{p} h\left(t, \tau ; \varphi_{n}^{*}(\tau)\right)\right)\right]\right]-\Phi\left[t ; \varphi(t) ; S_{\tau}\left[\frac{1}{\tau^{p}} U_{n}^{\tau}\left(\tau^{p} h(t, \tau ; \varphi(\tau))\right)\right]\right]\right\|_{\beta} \\
& \leq \mu_{8}\left(\mu_{1}+\mu_{2} \ln (2 n+1)\right)\left\{\left\|\Phi\left[t ; \varphi_{n}^{*}(t) ; S_{\tau}\left[\frac{1}{\tau^{p}} U_{n}^{\tau}\left(\tau^{p} h\left(t, \tau ; \varphi_{n}^{*}(\tau)\right)\right)\right]\right]-\Phi\left[t ; \varphi(t) ; S_{T}\left[\frac{1}{\tau^{p}} U_{n}^{\tau}\left(\tau^{p} h\left(t, \tau ; \varphi_{n}^{*}(\tau)\right)\right)\right]\right]\right\|_{\beta}\right. \\
& \left.+\left\|\Phi\left[t l \varphi(t) ; S_{T}\left[\frac{1}{\tau^{p}} U_{n}^{\tau}\left(\tau^{p} h\left(t, \tau ; \varphi_{n}^{*}(\tau)\right)\right)\right]\right]-\Phi\left[t ; \varphi(t) ; S_{T}\left[\frac{1}{\tau^{p}} U_{n}^{\tau}\left(\tau^{p} h(t, \tau ; \varphi(\tau))\right)\right]\right]\right\|_{\beta}\right\} \\
& \leq \mu_{8}\left(\mu_{1}+\mu_{2} \ln (2 n+1)\right)\left\{\left\|\Phi_{u}^{\prime}\left[t ; \xi_{n}^{(1)}(t) ; S_{T}\left[\frac{1}{\tau^{p}} U_{n}^{\tau}\left(\tau^{p} h\left(t, \tau ; \varphi_{n}^{*}(\tau)\right)\right)\right]\right]\right\|_{\beta}\left\|\varphi_{n}^{*}-\varphi\right\|_{\beta}\right. \\
& \left.+\left\|\Phi_{v}^{\prime}\left[t ; \varphi(t) ; S_{T}\left[\frac{1}{\tau^{p}} U_{n}^{T}\left(\tau^{p} h\left(t, \tau ; \xi_{n}^{(2)}(\tau)\right)\right)\right]\right]\right\|_{\beta}\left\|S_{\tau}\right\|_{\beta} \times \frac{1}{\min _{t \in \Gamma}|t|^{p}}\left\|U_{n}^{\tau}\right\|_{\beta} \max _{t \in \Gamma}|t|^{p}\left\|h_{u}^{\prime}\left(t, \tau ; \xi_{n}^{(2)}(\tau)\right)\right\|_{\beta}\left\|\varphi_{n}^{*}-\varphi\right\|_{\beta}\right\} \\
& \leq \frac{\mu_{10}+\mu_{11} \ln (2 n+1)+\mu_{13} \ln ^{2}(2 n+1)}{n^{r+\sigma(\alpha)-\beta}} H\left(\varphi^{(r)} ; \sigma(\alpha)\right) \leq r_{1}\left(1-q_{1}\right) .
\end{aligned}
$$

From the estimations (12), (13) and lemma (Krasnoselski, 1969), putting there $T=B_{n, p}$, we obtain that the equation $B_{n, p, \varphi_{n}}=U n \mathrm{f}$ has in the sphere $\left\|\psi_{n}-\varphi_{n}^{*}\right\|_{\beta} \leq r_{1}$ the unique solution $\varphi_{n}(t)$ and the estimation

$$
\frac{\rho_{n}}{1+q} \leq\left\|\varphi_{n}-\varphi_{n}^{*}\right\|_{\beta} \leq \frac{\rho_{n}}{1-q}
$$

is proved.

This means that for $n \geq n_{3}$ in some neighbourhood of $(2 \mathrm{n}+1)$-dimensional point $\left\{y_{k}\right\}_{k=-n}^{n}\left(y_{k}: \varphi_{n}^{*}(t)=\sum_{k=-n}^{n} y_{k} t^{k}\right)$ in the sphere $\left\|\Psi_{n}-\varphi_{n}^{*}\right\|_{\beta} \leq r_{1} \operatorname{SNE}(3)$ has the unique solution $\left\{c_{j}\right\}_{j=0}^{2 n}$.

For approximative solution (2) the following estimation

$$
\left\|\varphi_{n}^{*}-\varphi_{n}\right\|_{\beta} \leq \frac{\rho_{n}}{1-q} \leq \frac{\mu_{14}+\mu_{15} \ln (2 n+1)+\mu_{16}^{2} \ln (2 n+1)}{n^{r+\sigma(\alpha)-\beta}} H\left(\varphi^{(r)}, \sigma(\alpha)\right)
$$

is obtained.

From the last inequality, triangle inequality

$$
\left\|\varphi-\varphi_{n}\right\|_{\beta} \leq\left\|\varphi-\varphi_{n}^{*}\right\|_{\beta}+\left\|\varphi_{n}^{*}-\varphi_{n}\right\|_{\beta}
$$

and Lemma 6.1 (Zolotarevschi, 1991) the estimation (5) follows. The theorem 1 is proved.

2- Unimprovable estimations of convergence rate of quadratures methods for nonlinear SIE.

Theorem 1 establishes convergence of quadratures methods for solving nonlinear SIE in the case, when the equations are defined on closed smooth contour of complex plane. It turns out that if the contour satisfies stronger conditions on its smoothness, then in the convergence rate (5) the factor $\ln ^{2} n$ is substituted by $\ln n$ and this estimation becomes unimprovable.

Theorem 2 Let $\Gamma$ be a closed smooth of class $C(2, v)(0<v<1)(Z o l o t a r e v s c h i, 1996)$, i.e. $\psi^{\prime \prime}(t) \in H v(\Gamma)$. Moreover, all conditions of theorem 1 are fulfilled. Then all the assertions of theorem 1 are true with substitution of estimation of convergence rate (5) by the unimprovable estimate

$$
\left\|\varphi-\varphi_{n}\right\|_{\beta}=O\left(n^{-r+\beta-\sigma(\alpha)} \ln n\right) H\left(\varphi^{(r)}, \sigma(\alpha)\right) .
$$

Proof. Firstly we prove, that if $\Gamma$ belongs to the class $C(2 ; v)$ the Lipshitz condition (10) for the linear operator $B_{n, p}^{\prime}\left(\varphi_{n}^{0}\right)$ is fulfilled with less constant containing $n^{\beta} \ln (2 n+1)$ instead of $\ln ^{2}(2 n+1)$. As it is seen from inequality (12), this gives the possibility to extend the neighbourhood $\left\|\varphi_{n}-\varphi_{n}^{*}\right\|_{\beta} \leq r_{1}$ of the best uniform approximation of 
element $\varphi_{n}^{*} \in X_{n}(\Gamma)$, in which approximate solution $\varphi_{n}(t) \in X_{n}(\Gamma) \subset H_{\beta}(\Gamma)$ is looked for initial nonlinear SIE (1), using quadratures methods.

By virtue of theorem (4) from (Feras, 2009) for every function $g(t) \in C(\Gamma)$.

$$
\left\|g-U_{n} g\right\|_{L 2} \leq d_{1} E_{n}(g, \Gamma),
$$

where $d_{k}(k=1,2, \ldots)$ are completely determined constants not depending on $n, \operatorname{En}(g, \Gamma)$ is the best uniform approximation of function $g(t)$ of polynomial type (2) from $X n(\Gamma)$ and $L 2$ is the space of square integrable functions with norm

$$
\|g\|_{L 2}=\left(\frac{1}{l} \int_{\Gamma}|g(\tau)|^{2}|d \tau|\right)^{\frac{1}{2}} .
$$

Let now $Z_{n}^{(1)}$ and $Z_{n}^{(2)}$ be arbitrary fixed elements from $X_{n}(\Gamma)$. For any element $\psi_{n} \in X_{n}(\Gamma)$ estimate the value

$$
\begin{gathered}
\eta_{n} \stackrel{\text { def }}{=} \|\left[B _ { n , p } ^ { \prime } \left(z_{n}^{(1)}-B_{n, p}^{\prime}\left(z_{n, p}^{(2)}\right) \psi_{n}(t)\left\|_{\beta} \leq\right\| U_{n}\left\{\Phi_{n}^{\prime}\left[t ; z_{n}^{(1)}(t) ; S_{\tau}\left[\frac{1}{\tau^{p}} U_{n}^{\tau}\left(\tau^{p} h\left(t, \tau ; z_{n}^{(1)}(\tau)\right)\right)\right]\right]-\right.\right.\right. \\
\left.\Phi_{u}^{\prime}\left[t ; z_{n}^{(2)}(t) ; S_{\tau}\left[\frac{1}{\tau^{p}} U_{n}^{\tau}\left(\tau^{p} h\left(t, \tau ; z_{n}^{(2)}(\tau)\right)\right)\right]\right]\right\} \psi_{n}(t) \|_{\beta} \\
+\| U_{n}\left\{\Phi_{v}^{\prime}\left[t ; z_{n}^{(1)}(t) ; S_{\tau}\left[\frac{1}{\tau^{p}} U_{n}^{\tau}\left(\tau^{p} h\left(t, \tau ; z_{n}^{(1)}(\tau)\right)\right)\right]\right] S_{\tau}\left[\frac{1}{\tau^{p}} U_{n}^{\tau}\left(\tau^{p} h_{u}^{\prime}\left(t, \tau ; z_{n}^{(1)}(\tau)\right)\right) \psi_{n}(\tau)\right]-\right. \\
\left.\Phi_{v}^{\prime}\left[t ; z_{n}^{(2)}(t) ; S_{\tau}\left[\frac{1}{\tau^{p}} U_{n}^{\tau}\left(\tau^{p} h\left(t, \tau z_{n}^{(2)}(\tau)\right)\right)\right]\right] S_{\tau}\left[\frac{1}{\tau^{p}} U_{n}^{\tau}\left(\tau^{p} h_{u}^{\prime}\left(t, \tau ; z_{n}^{(2)}(\tau)\right)\right) \psi_{n}(\tau)\right]\right\} \|_{\beta}=\eta_{n}^{(1)}+\eta_{n}^{(2)} .
\end{gathered}
$$

Estimates for every term $\eta_{n}^{(1)}$ and $\eta_{n}^{(2)}$ are carried out separately. As it is shown in (Zolotarevschi, 1991), for every function $g(t) \in C(\Gamma)$ the next inequality is valid

$$
\left\|U_{n} g\right\|_{\beta} \leq d_{2} n^{\beta}\left\|U_{n} g\right\| c \leq d_{3} n^{\beta} \ln (2 n+1)\|g\| c .
$$

From here, taking into account (9), conditions 1) of theorem 1, and the well known formula of finite increments for operators, we get

$$
\begin{gathered}
\eta_{n}^{(1)} \leq\left\|U_{n} \Phi_{u}^{\prime}\left[t ; z_{n}^{(1)}(t) ; S_{\tau}\left[\frac{1}{\tau^{p}} U_{n}^{\tau}\left(\tau^{p} h\left(t, \tau ; z_{n}^{(1)}(\tau)\right)\right)\right]\right]-U_{n} \Phi_{u}^{\prime}\left[t ; z_{n}^{(2)}(t) ; S_{\tau}\left[\frac{1}{\tau^{p}} U_{n}^{\tau}\left(\tau^{p} h\left(t, \tau ; z_{n}^{(1)}(\tau)\right)\right)\right]\right]\right\|_{\beta}\left\|\psi_{n}\right\|_{\beta}+ \\
+\left\|U_{n}\left\{\left\{\Phi_{u}^{\prime}\left[t, z_{n}^{(2)}(t) ; S_{\tau}\left[\frac{1}{\tau^{p}} U_{n}^{\tau}\left(\tau^{p} h\left(t, \tau ; z_{n}^{(1)}(\tau)\right)\right)\right]\right]-\Phi_{u}^{\prime}\left[t, z_{n}^{(2)}(t) ; S_{\tau}\left[\frac{1}{\tau^{p}} U_{n}^{\tau}\left(\tau^{P} h\left(t, \tau ; z_{n}^{(2)}(\tau)\right)\right)\right]\right]\right\} \psi_{n}(t)\right\}\right\|_{\beta} \\
\left.\leq\left(\mu_{1}+\mu_{2} \ln (2 n+1)\right) d_{4}\left\|z_{n}^{(1)}-z_{n}^{(2)}\right\|_{\beta}\left\|\psi_{n}\right\|_{\beta}+d_{3} n^{\beta} \ln (2 n+1)\right) \| \Phi_{u}^{\prime}\left[t ; z_{n}^{(2)}(t) ; S_{\tau}\left[\frac{1}{\tau^{p}} U_{n}^{\tau}\left(\tau^{p} h\left(t, \tau ; z_{n}^{(1)}(\tau)\right)\right)\right]\right] \\
-\Phi_{u}^{\prime}\left[t ; z_{n}^{(2)}(t) ; S_{\tau}\left[\frac{1}{\tau^{p}} U_{n}^{\tau}\left(\tau^{p} h\left(t, \tau ; z_{n}^{(2)}(\tau)\right)\right)\right]\right]\|c\| \psi_{n}\left\|c \leq\left(d_{5}+d_{6} \ln (2 n+1)\right)\right\| U_{n} z_{n}^{(1)}-U_{n} z_{n}^{(2)}\left\|_{\beta}\right\| \psi_{n} \|_{\beta} \\
+d_{7} n^{\beta} \ln (2 n+1)\left\|S_{\tau}\left[\frac{1}{\tau^{p}} U_{n}^{\tau}\left(\tau^{p}\left[h\left(t, \tau ; z_{n}^{(1)}(\tau)\right)-h\left(t, \tau ; z_{n}^{(2)}(\tau)\right)\right]\right)\right]\right\| c .\left\|\psi_{n}\right\| c .
\end{gathered}
$$

Further, since $\frac{1}{\tau^{p}} U_{n}^{\tau}\left(\tau^{p}\left[h\left(t, \tau ; z_{n}^{(1)}(\tau)\right)-h\left(t, \tau ; z_{n}^{(2)}(\tau)\right)\right]\right)$ is a polynomial on degrees of $\tau$ and $\tau^{(-1)}$, then $S_{\tau}\left[\frac{1}{\tau^{p}} U_{n}^{\tau}\left(\tau^{p}\left[h\left(t, \tau ; z_{n}^{(1)}(\tau)\right)-h\left(t, \tau ; z_{n}^{(2)}(\tau)\right)\right]\right)\right]$ is also a polynomial and, hence, a continuous function on $\Gamma$.

\section{Results}

Therefore, using Holder inquality for integrals and estimate (Feras, 2006).

$$
\left\|U_{n}\right\|_{C \rightarrow L 2} \leq d_{9}<\infty
$$

Findings:

$$
\begin{gathered}
\left\|S_{\tau}\left[\frac{1}{\tau^{p}} U_{n}^{\tau}\left(\tau^{p}\left[h\left(t, \tau ; z_{n}^{(1)}(\tau)\right)-h\left(t, \tau ; z_{n}^{(2)}(\tau)\right)\right]\right)\right]\right\| c \leq d_{8}\left\|\frac{1}{\tau^{p}}\right\| l_{2} \| U_{n}^{\tau}\left(\tau^{p}\left[h\left(t, \tau ; z_{n}^{(1)}(\tau)-h\left(t, \tau ; z_{n}^{(2)}(\tau)\right)\right]\right) \| l_{2}\right. \\
\leq d_{8} \frac{1}{\left\|\tau^{p}\right\|_{L 2}}\left\|U_{n}^{\tau}\right\| c \rightarrow l_{2}\left\|\tau^{p}\right\| l_{2}\left\|\left(t, \tau ; z_{n}^{(1)}(\tau)\right)-h\left(t, \tau ; z_{n}^{(2)}(\tau)\right)\right\| l_{2}
\end{gathered}
$$




$$
\leq d_{8} d_{9} d_{10} \| h\left(t, \tau / z_{n}^{(1)}(\tau)-h\left(t, \tau ; z_{n}^{(2)}(\tau)\right)\left\|c \leq d_{11}\right\| z_{n}^{(1)}-z_{n}^{(2)} \| c .\right.
$$

From inequalities (16) and (18) taking acount of $\|\cdot\| c \leq\|\cdot\|_{\beta}$ for $\eta_{n}^{(1)}$, we finally get

$$
\eta_{n}^{(1)} \leq\left(d_{12}+d_{13} \ln (2 n+1)\right) n^{\beta}\left\|z_{n}^{(1)}-z_{n}^{(2)}\right\| c\left\|\psi_{n}\right\|_{\beta} .
$$

Estimate the quantity $\eta_{n}^{(2)}$

$$
\begin{gathered}
\eta_{n}^{(2)} \leq \| U_{n}\left\{\left(\Phi_{v}^{\prime}\left[t ; z_{n}^{(1)}(t) ; S_{\tau}\left[\frac{1}{\tau^{p}} U_{n}^{\tau}\left(\tau^{p} h\left(t, \tau ; z_{n}^{(1)}(\tau)\right)\right)\right]\right]\right.\right. \\
\left.\left.-\Phi_{v}^{\prime}\left[t ; z_{n}^{(2)}(t) ; S_{\tau}\left[\frac{1}{\tau^{p}} U_{n}^{\tau}\left(\tau^{p} h\left(t, \tau ; z_{n}^{(2)}(\tau)\right)\right)\right]\right]\right) S_{\tau}\left[\frac{1}{\tau^{p}} U_{n}^{\tau}\left(\tau^{p} h_{u}^{\prime}\left(t, \tau ; z_{n}^{(1)}(\tau)\right)\right) \psi_{u}(\tau)\right]\right\} \|_{\beta}+ \\
+\left\|U_{n}\left\{\Phi_{v}^{\prime}\left[t ; z_{n}^{(2)}(t) ; S_{\tau}\left[\frac{1}{\tau^{p}} U_{n}^{\tau}\left(\tau^{p} h\left(t, \tau ; z_{n}^{(2)}(\tau)\right)\right)\right]\right] \times\left(S_{\tau}\left[\frac{1}{\tau^{p}} U_{n}^{\tau}\left(\tau^{p}\left[h_{u}^{\prime}\left(t, \tau ; z_{n}^{(1)}(\tau)\right)-h_{u}^{\prime}\left(t, \tau ; z_{n}^{(2)}(\tau)\right)\right]\right) \psi_{n}(\tau)\right]\right)\right\}\right\|_{\beta} .
\end{gathered}
$$

As in the points $z_{n}^{(1)}$ and $z_{n}^{(2)}$ from $X_{n}(\Gamma)$ the inequality

$$
\begin{gathered}
\| S \tau\left[\frac{1}{\tau^{p}} U_{n}^{\tau}\left(\tau^{p} h_{u}^{\prime}\left(t, \tau ; z_{n}^{(1)}(\tau)\right)\right) \| \leq d_{14},\right. \\
\left\|\Phi_{v}^{\prime}\left[t ; z_{n}^{(2)}(t) ; S_{\tau}\left[\frac{1}{\tau^{p}} U_{n}^{\tau}\left(\tau^{p} h\left(t, \tau ; z_{n}^{(2)}(\tau)\right)\right)\right]\right]\right\|_{\beta} \leq d_{15}
\end{gathered}
$$

is satisfied, then carrying out analogous calculations, taking account of (19) and (16), we obtain

$$
\begin{gathered}
\eta_{n}^{(2)} \leq d_{14}\left(d_{15}+d_{16} \ln (2 n+1)\right) n^{\beta}\left\|z_{n}^{(1)}-z_{n}^{(2)}\right\|_{C}\|\psi\|_{\beta} \\
+d_{15} d_{3} n^{\beta} \ln (2 n+1)\left\|S_{\tau}\left[\frac{1}{\tau^{p}} U_{n}^{\tau}\left(\tau^{p}\left[h_{u}^{\prime}\left(t, \tau ; z_{n}^{(1)}(\tau)\right)-h_{u}^{\prime}\left(t, \tau ; z_{n}^{(2)}(\tau)\right)\right]\right)\right]\right\| c\left\|\psi_{n}\right\| c .
\end{gathered}
$$

Further by analogous reasonings, using Holder's inequality for integrals and estimate (17), we get

$$
\left\|S_{\tau}\left[\frac{1}{\tau^{p}} U_{n}^{\tau}\left(\tau^{p}\left[h_{u}^{\prime}\left(t, \tau ; z_{n}^{(1)}(\tau)\right)-h_{u}^{\prime}\left(t, \tau ; z_{n}^{(2)}(\tau)\right)\right]\right)\right]\right\| c \leq d_{17}\left\|z_{n}^{(1)}-z_{2}^{(2)}\right\| c .
$$

Hence, by virtue of (20) for $\eta_{n}^{(2)}$,it follows

$$
\eta_{n}^{(2)} \leq\left(d_{18}+d_{19} \ln (2 n+1)\right) n^{\beta}\left\|z_{n}^{(1)}-z_{n}^{(2)}\right\| c\|\psi\|_{\beta} .
$$

From (19) and (21) it follows that the linear operator $B_{n, p}^{\prime}\left(\varphi_{n}^{0}\right)$ satisfies Lipshitz condition with probably less constant

$$
\left\|B_{n, p}^{\prime}\left(z_{n}^{(1)}\right)-B_{n, p}^{\prime}\left(z_{n}^{(2)}\right)\right\|_{\beta} \leq\left(d_{20}+d_{21} \ln (2 n+1)\right) n^{\beta}\left\|z_{n}^{(1)}-z_{n}^{(2)}\right\| c .
$$

Further we estimate the quantity $\rho_{n}$ under condition that $\Gamma \in C(2, v)$. From (13) it is seen that for this it suffices to estimate the norm

$$
\left\|U_{n}\left\{\Phi\left[t ; \varphi_{n}^{*}(t) ; S_{\tau}\left[\frac{1}{\tau^{p}} U_{n}^{\tau}\left(\tau^{p} h\left(t, \tau ; \varphi_{n}^{*}(\tau)\right)\right)\right]\right]-\Phi\left[t ; \varphi(t) ; S_{\tau}\left[\frac{1}{\tau^{p}} U_{n}^{\tau}\left(\tau^{p} h\left(t, \tau ; \varphi_{n}^{*}(\tau)\right)\right)\right]\right]\right\}\right\|_{\beta}=\eta_{n}^{(3)}
$$

and ther norm

$$
\left\|U_{n}\left\{\Phi\left[t ; \varphi(\tau), S_{\tau}\left[\frac{1}{\tau^{p}} U_{n}^{\tau}\left(\tau^{p} h\left(t, \tau ; \varphi_{n}^{*}(\tau)\right)\right)\right]\right]-\Phi\left[t ; \varphi(t) ; S_{\tau}\left[\frac{1}{\tau^{p}} U_{n}^{\tau}\left(\tau^{P} h(t, \tau ; \varphi(\tau))\right)\right]\right]\right\}\right\|_{\beta}=\eta_{n}^{(4)}
$$

Using (16), formula of finite increments of operators, conditions 1) of theorem 1 and lemma 6.1 (Zolotarevschi, 1991) for $\eta_{n}^{(3)}$, we get

$$
\eta_{n}^{(3)} \leq d_{3} n^{\beta} \ln (2 n+1)\left\|\xi^{\prime}(t)\right\|_{c}\left\|\varphi^{*}-\varphi\right\| c \leq \frac{d_{23} \ln (2 n+1)}{n^{r+\sigma(\alpha)-\beta}} H\left(\varphi^{(r)} ; \sigma(\alpha)\right) .
$$

In order to estimate $\eta_{n}^{(4)}$, we use the some method we used to estimate the second term of $\eta_{n}^{(1)}$ with the help of (18). We obtain

$$
\eta_{n}^{(4)} \leq \frac{d_{24} \ln (2 n+1)}{n^{r+\sigma(\alpha)-\beta}} H\left(\varphi^{(r)} ; \sigma(\alpha)\right) .
$$


Hence with the condition $\Gamma \in C(2, . v)(0<v<1)$, the inequality (13) takes the form

$$
\rho_{n} \equiv\left\|\left[B_{n, p}^{\prime}\left(\varphi_{n}^{*}\right)^{-1}\right]\left[B_{n, p}\left(\varphi^{*}\right)-U_{n} f\right]\right\|_{\beta} \leq \frac{d_{25} \ln (2 n+1)}{n^{r+\sigma(\alpha)-\beta}} H\left(\varphi^{(r)} ; \sigma(\alpha)\right) \leq r_{1}(1-q)
$$

Further reasoning rare carried out analogously to that of the proof of theorem 1 . Theorem 2 is proved.

\section{References}

Feras, M. AL-Fagih. (2006). Approximate solution of singular integro-differential equations by reduction methods in generalized Holder spaces. World Scientific and Engineering Academy and Society (WSEAS), USA.

Feras, M. AL-Fagih. (2009). Function approximation in generalized holder spaces and numerical solution of singular integral equations. World Scientific and Engineering Academy and Society (WSEAS), USA.

Gabdullaev, B. G., \& Gorlov, V. E. (1976). Solving nonlinear singular integral equations by reduction method (Russian). Izv. vuzov., Matem., 2, pp. 3-13.

Gahov, F. D. (1977). Boundary - value problems (Russian). M.: Nauka.

Krasnoselski, M. A. (1969). Vainikko, G. M., Zabreiko, P. P., Rutitski, I. B., Stetsenko, B. Ia. Aproximate solution of operator equations (Russian). M.: Nauka.

Mushelishvili, N. I. (1968). Singular integral equations. M.: Nauka. English transl.: Noordhoff, 1972.

Scichiuc, V. N. (1984). On convergence of collocation method and method of mechanical guardratures for singular integral equations give on Lyapunov contour (Russian). Dif. yravnenia, 20(7), 1267-1275.

Seichiuc, V. N. (1997).Collocation method of solving nonlinear singular integral equations given on closed smooth contour. Buletinul Stiintific al Universitaii din Baia Mare, Seria B. Matematica - Informatica, 13(1-2), 147152.

Smirnov, V. M., \& Lebedev, N. A. (1964). Constructive theory of complex variable functions (Russian). M.-L.: Nauka.

Suetin, P. K. (1998). The series on Faber's polynomials. Gordan and Breach science Publisher.

Zolotarevschi, V. A. (1991). Finite dimensional methods of solving singular integral equations on closed contours of integration (Russian). Chishinau: Stiintsa.

Zolotarevschi, V. A. (1996). Direct methods for solving singular integral equations on closed smooth contour in spaces LP. Revue d'analise numerique et de theorie de l'approxoimation, Tome 25, No. 1-2, 257-265. 